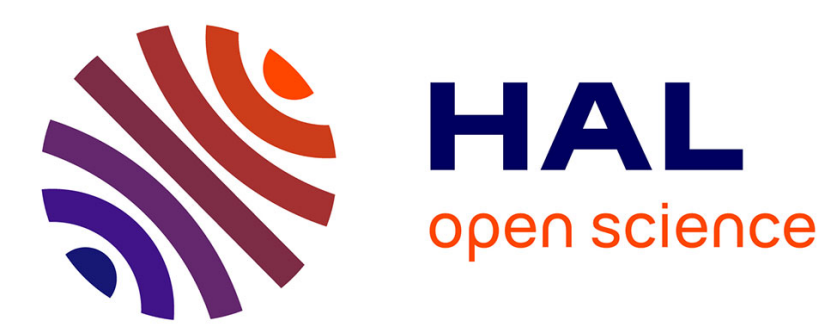

\title{
An extension of specificity-based approximations to other belief function relations
}

Tekwa Tedjini, Sohaib Afifi, Frédéric Pichon, Eric Lefèvre

\section{To cite this version:}

Tekwa Tedjini, Sohaib Afifi, Frédéric Pichon, Eric Lefèvre. An extension of specificity-based approximations to other belief function relations. 6th International Conference on Belief Functions, Sep 2021, Shanghai, China. pp.224-233. hal-03520319

\section{HAL Id: hal-03520319 \\ https://hal.science/hal-03520319}

Submitted on 11 Jan 2022

HAL is a multi-disciplinary open access archive for the deposit and dissemination of scientific research documents, whether they are published or not. The documents may come from teaching and research institutions in France or abroad, or from public or private research centers.
L'archive ouverte pluridisciplinaire HAL, est destinée au dépôt et à la diffusion de documents scientifiques de niveau recherche, publiés ou non, émanant des établissements d'enseignement et de recherche français ou étrangers, des laboratoires publics ou privés. 


\title{
An extension of specificity-based approximations to other belief function relations
}

\author{
Tekwa Tedjini ${ }^{1}$, Sohaib Afifi ${ }^{1}$, Frédéric Pichon $^{1}$, and Eric Lefèvre ${ }^{1}$ \\ Univ. Artois, UR 3926, Laboratoire de Genie Informatique et d'Automatique de l'Artois \\ (LGI2A), F-62400 Béthune, France \\ firstname.lastname@univ-artois.fr
}

\begin{abstract}
Adopting a general framework to faithfully represent uncertainty, such as belief function theory, usually comes at a cost. In many real-life applications, we are constrained to handle mass functions that have too many focal elements. Fortunately, one can resort to approximation techniques to bypass this issue. In this paper, we extend the classical approximation techniques, which are mainly specificity-based, to other belief function relations such as lattice dominance. This allows to overcome the limits of classical techniques in some applications.
\end{abstract}

Keywords: Belief function $\cdot$ Approximation $\cdot$ Specificity $\cdot$ Relations.

\section{Introduction}

Belief function theory [15] is a rich and powerful uncertainty reasoning framework as it extends both the set and probability representations of uncertainty. Despite its successful application in many real-life problems, it has been criticized for its high computational complexity. Several techniques have been proposed to simplify the computations pertaining to this theory, either using exact [12] or approximate methods. We are particularly interested in the latter. Approximations can be computed by Monte-Carlo simulations [20], or by replacing the original mass function by a probability measure or a possibilistic one [19,7]. Other approaches can be used where mass functions are combined on a coarsened frame of discernment [3] or where the number of focal sets is reduced $[18,1,13,8,14,2]$. We draw a particular attention to this last family of methods. Besides simplicity, a good approximation has to be consistent and close enough to the original mass function [8]. Closeness is typically quantified by a distance measure, whereas consistency is unanimously based on comparing the specificity of the informative content of the original mass function and its approximation. Recently, Destercke and al. [4] introduced an approach that extends any set relation to belief functions. This approach generalizes the notion of comparison and allows, along with comparing the informative content of beliefs in terms of specificity, to establish other relations between them such as dominance. In this paper, we propose to extend this approach to approximation methods that reduce the number of focal sets of mass functions. We are motivated by the deficiency of classical approximation techniques in some applications. This deficiency arises from the use of approximate beliefs that are more or less specific than the original ones whilst the application requires rather to choose beliefs that are, for instance, dominant. We will develop this idea later in the paper. 
The remainder of this paper is organized as follows. Section 2 gives a quick reminder on belief functions and set relations. Section 3 describes the notion of comparison in belief function theory. The proposed generalized approximation and a particular case study are presented in section 4 . We conclude the paper in Section 5.

\section{Basic definitions}

In this section, we provide some basic definitions on belief functions and set relations that are required in our developments.

\subsection{Theory of belief functions}

Let $x$ be an uncertain variable defined on finite set of values $\mathcal{X}=\left\{x_{1}, x_{2}, \ldots, x_{n}\right\}$ called the frame of discernment. The available knowledge about $x$ is represented by a mass function $m^{\mathcal{X}}: 2^{\mathcal{X}} \mapsto[0,1]$ s.t. $\sum_{A \subset \mathcal{X}} m^{\mathcal{X}}(A)=1$ and $m^{\mathcal{X}}(\emptyset)=0 . m^{\mathcal{X}}(A)$ quantifies the part of our belief that $x \in A$ without providing any further information about $x \in A^{\prime} \subset A$. Each subset $A \subseteq \mathcal{X}$ such that $m^{\mathcal{X}}(A)>0$ is called focal set or focal element of $m^{\mathcal{X}}$. Other knowledge representations can be obtained from $m^{\mathcal{X}}$, such as the belief $\mathrm{Bel}^{\mathcal{X}}$ and the plausibility $\mathrm{Pl}^{\mathcal{X}}$ function, defined for all $A \subseteq \mathcal{X}$ :

$$
B e l^{\mathcal{X}}(A)=\sum_{\emptyset \neq B \subseteq A} m^{\mathcal{X}}(B), \quad P l^{\mathcal{X}}(A)=\sum_{B \cap A \neq \emptyset} m^{\mathcal{X}}(B) .
$$

$B e l^{\mathcal{X}}$ is the amount of evidence that supports $x \in A$ and $P l^{\mathcal{X}}$ is interpreted as the amount of evidence that is consistent with $x \in A$.

\subsection{Set relations}

A relation $\mathbf{R}$ between subsets of $\mathcal{X}$ is a subset $\mathbf{R} \subseteq 2^{\mathcal{X}} \times 2^{\mathcal{X}}$ that specifies which pair of subsets are related to each other [4]. Let $A$ and $B$ be two subsets of $\mathcal{X}$. We denote by $A \mathbf{R} B$ whenever $(A, B) \in \mathbf{R}$. A relation may have several properties such as: reflexivity ( $A \mathbf{R} A, \forall A \subseteq \mathcal{X}$ ), transitivity ( $A \mathbf{R} B$ and $B \mathbf{R} C \Rightarrow A \mathbf{R} C$, with $C \subseteq \mathcal{X}$ ), antisymmetry ( $A \mathbf{R} B \wedge B \mathbf{R} A \Rightarrow A=B, \forall A, B \subseteq \mathcal{X}$ ), etc. Note that it is also possible to define more complex relations by combining those properties. For instance, the set-inclusion relation ( $A \mathbf{R} B \Leftrightarrow A \subseteq B$ ) is reflexive, transitive and antisymmetric [4].

\section{Comparing belief structures}

According to the Least Commitment Principle [16], if we have to choose among multiple mass functions compatible with a set of constraints, the most appropriate one is the least informative. To use this principle, one has to define tools to compare the content of the available mass functions. This is commonly done via the notion of specialization [6]. Given two mass functions $m_{1}^{\mathcal{X}}$ and $m_{2}^{\mathcal{X}}$ defined on $\mathcal{X}, m_{1}^{\mathcal{X}}$ is said to be at least as informative (specific) as $m_{2}^{\mathcal{X}}$, which we denote by $m_{1}^{\mathcal{X}} \sqsubseteq m_{2}^{\mathcal{X}}$, if and only if $m_{1}^{\mathcal{X}}$ can be obtained from $m_{2}^{\mathcal{X}}$ by sharing each mass $m_{2}^{\mathcal{X}}(B)$ among subsets $A \subseteq B$. 
Formally, there exists a non-negative square matrix, known as the specialization matrix $S=[S(A, B)], A, B \in 2^{\mathcal{X}}$, verifying the conditions below:

$$
\begin{gathered}
\sum_{A \subseteq \mathcal{X}} S(A, B)=1, \quad \forall B \subseteq \mathcal{X}, \\
S(A, B)>0 \Rightarrow A \subseteq B, \quad \forall A, B \subseteq \mathcal{X}, \\
m_{1}^{\mathcal{X}}(A)=\sum_{B \subseteq \mathcal{X}} S(A, B) m_{2}^{\mathcal{X}}(B), \quad \forall A \subseteq \mathcal{X} .
\end{gathered}
$$

$S(A, B) \in[0,1]$ is the proportion of $m_{2}^{\mathcal{X}}(B)$ that flows into $A \subseteq B$. Note that if $m_{1}^{\mathcal{X}} \sqsubseteq m_{2}^{\mathcal{X}}$ then [6]:

$$
\left[B e l_{1}^{\mathcal{X}}, P l_{1}^{\mathcal{X}}\right] \subseteq\left[B e l_{2}^{\mathcal{X}}, P l_{2}^{\mathcal{X}}\right] .
$$

The recent work of Destercke and al. [4] highlighted the relevance of investigating other links, besides specificity, between mass functions, particularly those extending set relations such as equivalence or partial/total order. The authors introduced a more general definition of the comparison of belief function as follows:

Definition 1. Let $m_{1}^{\mathcal{X}}$ and $m_{2}^{\mathcal{X}}$ be two mass functions and let $\mathbf{R}$ be a relation between subsets of $\mathcal{X}$. We say that $m_{1}^{\mathcal{X}} \widetilde{\mathbf{R}} m_{2}^{\mathcal{X}}$ if there is a left stochastic matrix $S$, such that $\forall A, B \subseteq \mathcal{X}$.

$$
\begin{gathered}
m_{1}^{\mathcal{X}}(A)=\sum_{B \subseteq \mathcal{X}} S(A, B) m_{2}^{\mathcal{X}}(B), \\
(S(A, B)>0) \wedge\left(m_{2}^{\mathcal{X}}(B)>0\right) \Rightarrow A \mathbf{R} B .
\end{gathered}
$$

$S(A, B)$ is the proportion of $m_{2}^{\mathcal{X}}(B)$ transferred to $A$, such that $A \mathbf{R} B$ [4].

Note that when $\widetilde{\mathbf{R}}$ is replaced by $\sqsubseteq$ and $\mathbf{R}$ by $\subseteq$ in (7), we obtain the specialization relation defined earlier. Furthermore, when $\mathcal{X}$ is ordered, it is also possible to recover another relation that was studied in [9], by comparing two subsets $A, B \subseteq \mathcal{X}$ defined as $A=\{\underline{a}, \ldots, \bar{a}\}(\underline{a} \leq \bar{a})$ and $B=\{\underline{b}, \ldots, \bar{b}\}(\underline{b} \leq \bar{b})$ in terms of lattice dominance [4]. We say then that $m_{1}^{\mathcal{X}}$ is at least as small as $m_{2}^{\mathcal{X}}$, which we denote by $m_{1}^{\mathcal{X}} \preceq m_{2}^{\mathcal{X}}$, with $\widetilde{\mathbf{R}}$ being replaced by $\preceq$ and $\mathbf{R}$ replaced by $\leq_{d}$ where $A \leq_{d} B$ if $\underline{a} \leq \underline{b}$ and $\bar{a} \leq \bar{b}$. The following property holds [9]:

$$
m_{1}^{\mathcal{X}} \preceq m_{2}^{\mathcal{X}} \Rightarrow\left[\mathrm{Bel}_{2}^{\mathcal{X}}, P l_{2}^{\mathcal{X}}\right] \leq_{d}\left[\mathrm{Bel}_{1}^{\mathcal{X}}, P l_{1}^{\mathcal{X}}\right] .
$$

\section{Generalization of belief functions approximation}

Usually, a mass function $m$ is approximated by another mass function $m^{\prime}$ that is at most as specific as $m$, i.e., $m \sqsubseteq m^{\prime}$. Assume that we want to approximate $m$ by reducing the number of its focal sets. $m^{\prime}$ can be built from $m$ by preserving the most significant focal sets, i.e., those with high mass values, and by aggregating or removing the redundant or the least significant ones as in [13]. It is also possible to reduce the number of focal sets iteratively as in $[8,14,2]$. These latter methods help to trade-off between the quality and the computational time required to determine $m^{\prime}$. 
In this section, we extend the previously stated techniques to other possible relations $\tilde{\mathbf{R}}$ between $m$ and $m^{\prime}$. Our motivation arises from the fact that specificity-based approximations may be inappropriate in some applications, such as in the combinatorial optimization problem that we studied in [17]. Specifically, we proposed in [17] a belief-constrained programming approach inspired from [9] to model the vehicle routing problem with time windows [11] and evidential service and travel times. In this kind of problems, each vehicle is compelled to start the service at any customer within his time availability interval (window). Arrivals after the closure of time windows are therefore forbidden. To fulfill such particular constraints, given the evidential time parameters, confidence levels are imposed on the belief and the plausibility functions of the arrival times which are combination of service and travel times. For instance, if $x$ is the variable representing the arrival time at a given customer, $C$ is the closure of his time window and $\alpha, \beta \in[0,1](\alpha \leq \beta)$ are two confidence levels, the time constraints for this customer can be expressed as:

$$
\operatorname{Bel}(x \leq C) \geq \alpha, \quad P l(x \leq C) \geq \beta .
$$

The use of belief functions adds more complexity to the problem that is already NPhard. The problem involves indeed costly mass function combinations due to large numbers of focal sets. Consequently, we turned to classical approximation methods to overcome this issue. Nevertheless, we noticed that replacing the original service and travel time mass functions by less specific ones impacts inappropriately the set of feasible solutions, i.e., solutions that satisfy all the problem constraints. Indeed, a solution may be feasible when using approximations while it is rejected when using the original mass functions. Take for instance the variable $x$ defined earlier, and suppose that uncertainty about the value of $x$ is represented by the mass function: $m(\{15,16\})=0.9$, $m(\{16,17\})=0.05, m(\{16.30,17.30\})=0.05$. Suppose that $C=16$ and that $\alpha=0.9, \beta=1$. Using (1), we have $\operatorname{Bel}(x \leq 16)=0.9=\alpha$ and $P l(x \leq 16)=$ $0.95<\beta$. The confidence level $\beta$ is not met, thus the customer can not be served. Suppose now that uncertainty about $x$ is represented using an approximation $m^{\prime}$ such that $m \sqsubseteq m^{\prime} . m^{\prime}$ is given by $m^{\prime}(\{15,16\})=0.9, m^{\prime}(\{16,16.30,17,17.30\})=0.1$. We have $\operatorname{Bel}^{\prime}(x \leq 16)=0.9=\alpha$ and $P l^{\prime}(x \leq 16)=1=\beta$. Note that $B e l^{\prime}=B e l$ and $P l^{\prime}>P l$, this is due to the relation in (5). In this case, both of the confidence levels are verified and the customer in question can be served. Such a result is quite contradictory with the information we had originally. Hence, it is worthwhile to introduce a more general approach so that one can properly approximate a mass function by another one that is more/less specific or smaller/greater or equivalent, etc, to span a broad range of real-life applications.

\subsection{Formalization}

Building on the formal definition of approximations given in [2], we can introduce a generalized definition of an approximation as follows:

Definition 2. Let $\mathcal{P}=\left\{P_{1}, P_{2}, \ldots, P_{K}\right\}$ be a partition of the set $\mathbb{N}_{n}=\{1, \ldots, n\}$, i.e., $P_{k} \cap P_{l}=\emptyset$ and $\bigcup_{k=1}^{K} P_{k}=\mathbb{N}_{n}$ and let $m$ be a mass function with focal elements 
$\mathcal{F}(m)=\left\{A_{1}, A_{2}, \ldots, A_{n}\right\}$ such that $m\left(A_{i}\right) \geq m\left(A_{i+1}\right), \forall i=1, \ldots, n-1$. Let $m^{\prime}$ be another mass function with $\mathcal{F}\left(m^{\prime}\right)=\left\{B_{1}, \ldots, B_{K}\right\}$ its focal sets verifying for each $k=1, \ldots, K$ :

$$
\begin{gathered}
A_{i} \mathbf{R} B_{k}, \forall i \in P_{k}, \\
m^{\prime}\left(B_{k}\right)=\sum_{i \in P_{k}} m\left(A_{i}\right) .
\end{gathered}
$$

$m^{\prime}$ is called $\widetilde{\mathbf{R}}$-approximation of $m$.

Definition 2 states that for a given relation $\tilde{\mathbf{R}}$, any mass function $m^{\prime}$ with fewer focal sets and that is related to $m$ by $\tilde{\mathbf{R}}$, i.e., $m \tilde{\mathbf{R}} m^{\prime}$, is an approximation of $m$. Note that $m$ and $m^{\prime}$ verify the conditions of Definition 1 as it is possible, for any $P_{k} \in \mathcal{P}(k=$ $1, \ldots, K)$, to retrieve $m\left(A_{i}\right)$ from $m^{\prime}\left(B_{k}\right)$ by transferring a proportion $S\left(A_{i}, B_{k}\right)>0$ of the mass $m^{\prime}\left(B_{k}\right)>0$ from the subset $B_{k}$ to the subset $A_{i}$ such that $A_{i} \mathbf{R} B_{k}$, with :

$$
S\left(A_{i}, B_{k}\right)=\frac{m\left(A_{i}\right)}{m^{\prime}\left(B_{k}\right)}=\frac{m\left(A_{i}\right)}{\sum_{j \in P_{k}} m\left(A_{j}\right)}
$$

Particular cases: Definition 2 covers some well known cases that were already studied in the literature. For instance, if $\mathbf{R}$ is an outer-inclusion relation, i.e., $A_{i} \subseteq B_{k}$, with $B_{k}=\bigcup_{i \in P_{k}} A_{i}$, then $\widetilde{\mathbf{R}}=\sqsubseteq$, that is $m \sqsubseteq m^{\prime}$, which corresponds to the outer approximations of the literature $[2,14,8,13]$.

We can also identify another sub-case when $\mathbf{R}$ is an inner-inclusion relation, i.e., $A_{i} \supseteq$ $B_{k}$, where $B_{k}=\bigcap_{i \in P_{k}} A_{i}$. In this case $\widetilde{\mathbf{R}}=\sqsupseteq$ that is $m \sqsupseteq m^{\prime}$, which is the inner approximation of Denœux [2].

Furthermore, if an order is established on $\mathcal{X}$ and $\widetilde{\mathbf{R}}=\preceq$, it is also possible to approximate $m$ by a mass function $m^{\prime}$ such that $m \preceq m^{\prime}$, where $\preceq$ is the generalized lattice dominance relation. This new approximation is detailed in Section 4.2.

To use the generalized approximation, one can for instance keep the first $K-1$ most significant focal sets of $m$ and replace the remaining focal sets by a set $B$ such that $A_{i} \mathbf{R} B, \forall i=K, \ldots, n$. This is the generalization of the summarization [13]. However, to provide a good quality approximation, we propose to combine the summarization with the hierarchical clustering procedure introduced in [2]. The main idea of our procedure is to preserve the first (most significant) $p$ focal sets $(p<K<n)$, then reduce iteratively, starting from $p+1$, the number of the remaining focal sets, i.e., those with relatively small masses. At each iteration, a similarity measure or a distance is computed between each pair of focal sets $A_{i}$ and $A_{j}$, then the most similar/nearest pair $\left(A_{i^{*}}, A_{j^{*}}\right)$ is replaced by a set $B_{i t e r}$, such that $A_{i^{*}} \mathbf{R} B_{i t e r}$ and $A_{j^{*}} \mathbf{R} B_{\text {iter }}$ with $B_{\text {iter }}$ being similar to $A_{i^{*}}$ and $A_{j^{*}}$, and where $m\left(B_{\text {iter }}\right)=m\left(A_{i^{*}}\right)+m\left(A_{j^{*}}\right)$. The process is repeated until we reach size $K$. The pseudo-code of the approach is explained in Algorithm 1 which runs in a time complexity of $\mathcal{O}\left(n^{3}\right)$. The worst case number of iterations in the repeat loop is $(n-1)$ and the most expensive instruction inside this loop is the update of the similarity matrix $\mathcal{O}\left(n^{2}\right)$, this yields a total complexity of $\mathcal{O}\left(n^{3}\right)$. 


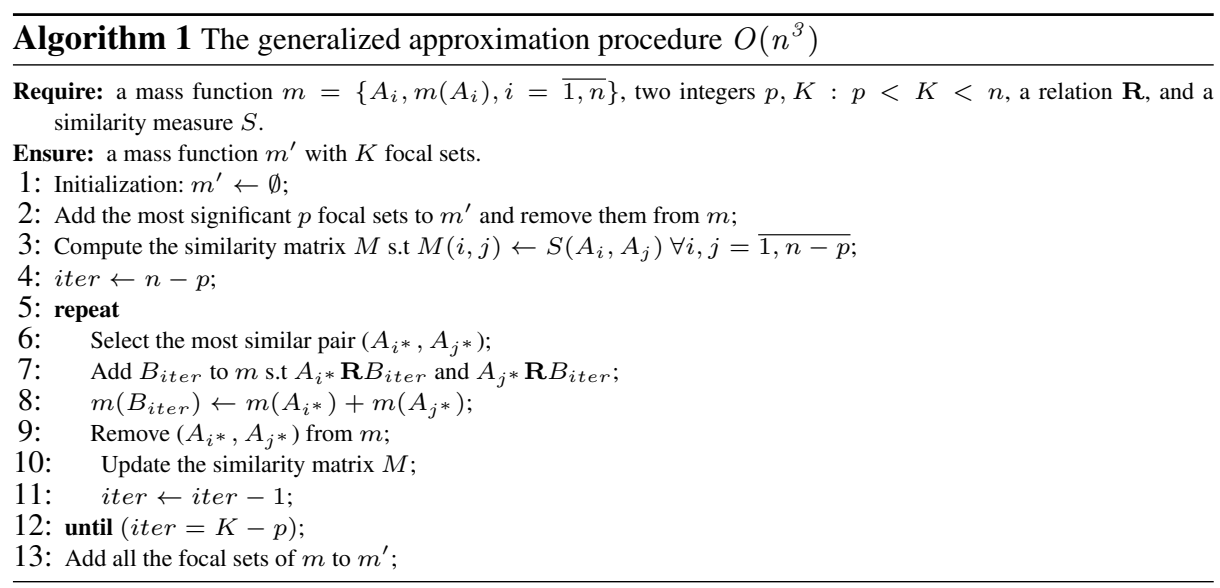

\subsection{A lattice dominance-based approximation}

This section studies a particular case of the general approximation where $\widetilde{\mathbf{R}}=\preceq$. In other words, we want to approximate $m$ with a mass function $m^{\prime}$ that is greater than $m$ according to lattice dominance. Note that property (8) holds with this partial order relation.

Consider an ordered set $\mathcal{X}=\left\{x_{1}, \ldots, x_{n}\right\}\left(x_{1} \leq \ldots \leq x_{n}\right)$ and a mass function $m$ defined on $\mathcal{X}$ having the following focal sets $\overline{\mathcal{F}}(m)=\left\{A_{1}, \ldots, A_{n}\right\}$ s.t $A_{i}=$ $\left\{\underline{a}_{i}, \ldots, \bar{a}_{i}\right\}\left(\underline{a}_{i} \leq \bar{a}_{i}\right)$, which we denote by $\llbracket \underline{a}_{i}, \bar{a}_{i} \rrbracket$, and where $m\left(A_{i}\right) \geq m\left(A_{i+1}\right), \forall i=$ $1, \ldots, n-1$. Using Definition 2, we can build a lattice dominance-based approximation (々-approximation) $m^{\prime}$ of $m$ such that $m \preceq m^{\prime}$ and where focal sets of $m^{\prime}$ are the subsets $B_{k}=\llbracket \underline{b}_{k}, \bar{b}_{k} \rrbracket=\left\{\underline{b}_{k}, \ldots, \overline{b_{k}}\right\}$, with $\underline{b}_{k} \leq \bar{b}_{k}$, and verifying for each $i \in P_{k}$ and $k=1, \ldots, K, A_{i} \leq_{d} B_{k}$, i.e., $\underline{a}_{i} \leq \underline{b}_{k}$ and $\bar{a}_{i} \leq \bar{b}_{k}$.

To illustrate this approximation, we use Algorithm 1 with the lattice dominance relation $\leq_{d}$ and Jaccard's similarity measure given by: $S_{\text {Jaccard }}\left(A_{i}, A_{j}\right)=\frac{\left|A_{i} \cap A_{j}\right|}{\left|A_{i} \cup A_{j}\right|}[10]$. The pair of the most similar focal sets $\left(A_{i^{*}}, A_{j^{*}}\right)$ is replaced by the subset $B_{i t e r}$ that is the nearest to $A_{i^{*}}$ and $A_{j^{*}}$ and which is defined as follows:

$$
B_{i t e r}=\llbracket \max \left(\underline{a}_{i^{*}}, \underline{a}_{j^{*}}\right), \max \left(\bar{a}_{i^{*}}, \bar{a}_{j^{*}}\right) \rrbracket
$$

The process is repeated until $m^{\prime}$ reaches size $K$. Note that the choice of an adequate measure depends on the relation that is used as well as the application in hand. Jaccard's measure can be replaced by any other similarity measure such as Dice's measure [5] or others. Moreover, if $m$ has disjoint focal sets, one can use a geometric distance [21] instead to capture the nearest focal sets.

Example 1. Let us use Algorithm 1 to build a $\preceq$-approximation for the mass function $m$ defined such that: $\mathcal{F}(m)=\left\{A_{1}=\llbracket 1,3 \rrbracket, A_{2}=\llbracket 2,7 \rrbracket, A_{3}=\llbracket 3,9 \rrbracket, A_{4}=\llbracket 1,6 \rrbracket, A_{5}=\right.$ $\left.\llbracket 6,8 \rrbracket, A_{6}=\llbracket 2,4 \rrbracket\right\}$ with $m\left(A_{1}\right)=0.4, m\left(A_{2}\right)=0.3, m\left(A_{3}\right)=0.1, m\left(A_{4}\right)=$ $0.1, m\left(A_{5}\right)=0.05, m\left(A_{6}\right)=0.05$. Also let $K=4$ and $p=2$.

* Step 1: Add $A_{1}$ and $A_{2}$ to $m^{\prime}$, then remove them from $m$. In this case, $m$ becomes: 
$\mathcal{F}(m)=\left\{A_{3}=\llbracket 3,9 \rrbracket, A_{4}=\llbracket 1,6 \rrbracket, A_{5}=\llbracket 6,8 \rrbracket, A_{6}=\llbracket 2,4 \rrbracket\right\}$ with $m\left(A_{3}\right)=$ $0.1, m\left(A_{4}\right)=0.1, m\left(A_{5}\right)=0.05, m\left(A_{6}\right)=0.05$.

* Step 2: Compute the similarity matrix $M$ for $m$, iter $=n-p=4$.

\begin{tabular}{|l|llll|}
\hline F. sets & $A_{3}$ & $A_{4}$ & $A_{5}$ & $A_{6} \star$ \\
\hline$A_{3}$ & - & 0.44 & 0.43 & 0.25 \\
$A_{4} \star$ & - & - & 0.13 & $\mathbf{0 . 5 0}$ \\
$A_{5}$ & - & - & - & 0 \\
$A_{6}$ & - & - & - & - \\
\hline
\end{tabular}

The most similar pair of focal sets $\left(A_{4}, A_{6}\right)$ is replaced, in $m$, by the subset $B_{i t e r}=B_{4}$ $=\llbracket 2,6 \rrbracket$ that is computed using equation (13), and $m\left(B_{4}\right)=m\left(A_{4}\right)+m\left(A_{6}\right)=0.15$. Hence $m$ becomes: $\mathcal{F}(m)=\left\{A_{3}=\llbracket 3,9 \rrbracket, B_{4}=\llbracket 2,6 \rrbracket, A_{5}=\llbracket 6,8 \rrbracket\right\}$ with $m\left(A_{3}\right)=$ $0.1, m\left(B_{4}\right)=0.15$ and $m\left(A_{5}\right)=0.05$.

* Step 3: Update the similarity matrix $M$, iter $=$ iter $-1=3$.

\begin{tabular}{|l|lll|}
\hline F. sets & $A_{3}$ & $B_{4 \star}$ & $A_{5}$ \\
\hline$A_{3} \star$ & - & $\mathbf{0 . 5 0}$ & 0.43 \\
$B_{4}$ & - & - & 0.14 \\
$A_{5}$ & - & - & - \\
\hline
\end{tabular}

The pair $\left(A_{3}, B_{4}\right)$ is replaced, in $m$, by the subset $B_{i t e r}=B_{3}=\llbracket 3,9 \rrbracket$, given (13), and $m\left(B_{3}\right)=m\left(A_{3}\right)+m\left(B_{4}\right)=0.25$. Hence $m$ becomes: $\mathcal{F}(m)=\left\{B_{3}=\llbracket 3,9 \rrbracket, A_{5}=\right.$ $\llbracket 6,8 \rrbracket\}$, with $m\left(B_{3}\right)=0.25$ and $m\left(A_{5}\right)=0.05$.

* Step 4: iter $=$ iter $-1=2=K-p$ : Stop and add $B_{3}$ and $A_{5}$ to $m^{\prime}$.

$m^{\prime}$ is the $\preceq$-approximation of $m$ where $\mathcal{F}\left(m^{\prime}\right)=\left\{A_{1}=\llbracket 1,3 \rrbracket, A_{2}=\llbracket 2,7 \rrbracket, B_{3}=\right.$ $\left.\llbracket 3,9 \rrbracket, A_{5}=\llbracket 6,8 \rrbracket\right\}$, with $m^{\prime}\left(A_{1}\right)=0.4, m^{\prime}\left(A_{2}\right)=0.3, m^{\prime}\left(B_{3}\right)=0.25$ and $m^{\prime}\left(A_{5}\right)=0.05$.

\subsection{Preliminary tests:}

The lattice dominance-based approximation method was incorporated within a metaheuristic framework to accelerate the solution scheme of the combinatorial optimization problem studied in [17], while preventing the increase of the set of feasible solutions. Tests were conducted on an adaptation of medium to large-sized literature instances. The details about the solution scheme as well as the instances adaptation are explained in [17]. Table 1 presents average cost results for instances Inst of 50 customers, after performing 15 executions per instance. Note that the meta-heuristic algorithm stops after 50 iterations without improvement. Columns 2 (resp. 4) and 3 (resp. 5) show costs $C$ (resp. $C_{\preceq}$ ) without (resp. with) $\preceq$-approximation and the corresponding execution time $C P U(s)$ (resp. $C P U_{\preceq}(s)$ ) recorded in seconds. The percentage of increase in solution cost induced by $\preceq$-approximation is displayed in column 6. Average costs $C_{\preceq}^{*}$ of solutions using $\preceq$-approximation for the same amount of time as in column 3 are presented in column 7. The experiments show a significant decrease in $C P U_{\preceq}(s)$ when using the approximation, this is expected since the number of focal sets is reduced. Moreover, the increase in cost values when using approximation is around $7.18 \%$ which is quite acceptable given the gain in time. In addition, the highlighted costs in column 7 , confirm that incorporating the $\preceq$-approximation in the meta-heuristic scheme helps 
to enhance the solution quality. Specifically, providing fast solutions helps the metaheuristic engine to explore, rapidly, further regions of the set of feasible solutions that might contain better quality solutions. Note that we chose to present results on mediumsized instances to highlight the advantage of using the proposed approximation as we were not even able to get results without approximation for large scale instances.

Table 1: Comparing results with and without $\prec$-approximation.

\begin{tabular}{rrrrrrr}
\hline Inst & $C$ & $C P U(s)$ & $C_{\preceq}$ & $C P U_{\preceq}(s)$ & Inc $(\%)$ & $C_{\preceq}^{*}$ \\
\hline C102 & 7549.88 & 260.70 & 7770.40 & 26.20 & 2.92 & $\mathbf{7 2 3 8 . 4 1}$ \\
C104 & $\mathbf{6 0 5 2 . 4 5}$ & 668.50 & 6391.06 & 48.77 & 5.59 & 6421.58 \\
C204 & 3580.75 & 356.71 & 3671.60 & 61.00 & 2.55 & $\mathbf{3 4 7 4 . 2 6}$ \\
R104 & $\mathbf{1 0 4 7 9 . 6 0}$ & 970.83 & 11947.63 & 27.50 & 9.55 & 11573.78 \\
R204 & 4026.19 & 742.13 & 4509.74 & 66.50 & 12.01 & $\mathbf{3 8 4 2 . 1 9}$ \\
R207 & 5246.60 & 299.40 & 5484.06 & 44.80 & 4.52 & $\mathbf{4 2 9 4 . 7 9}$ \\
R208 & $\mathbf{3 3 9 9 . 4 3}$ & 1512.27 & 3734.58 & 70.50 & 9.85 & 3411.02 \\
RC204 & 4368.41 & 494.40 & 4827.95 & 60.10 & 10.51 & $\mathbf{3 8 7 4 . 1 2}$ \\
\hline
\end{tabular}

\section{Conclusions and perspectives}

We proposed a general approach to approximate belief functions. This approach benefits from the generalization of set relations to belief functions and offers to simplify a mass function given any possible relation with its approximation. The presented approach includes some well known sub-cases, such that the inner and outer approximations of the literature. A lattice dominance-based case study was detailed and applied to a combinatorial optimization problem to accelerate the solution search. In future work, we will investigate other possible relations as well as the definition of other similarity measures that are problem-related to get more efficient results. An extension to approximations that are concerned with reducing the size of the frame of discernment is also an interesting perspective.

\section{References}

1. Bauer, M.: Approximation algorithms and decision making in the Dempster-Shafer theory of evidence -An empirical study. Int J Approx Reason 17(2-3), 217-237 (1997)

2. Denœux, T.: Inner and outer approximation of belief structures using a hierarchical clustering approach. Int J Uncertain Fuzz 9(4), 437-460 (2001)

3. Denœux, T., Yaghlane, A.B.: Approximating the combination of belief functions using the fast Möbius transform in a coarsened frame. Int J Approx Reason 31(1), 77 - 101 (2002)

4. Destercke, S., Pichon, F., Klein, J.: From set relations to belief function relations. Int J Approx Reason 110, 46-63 (2019)

5. Dice, L.R.: Measures of the amount of ecologic association between species. Ecology 26(3), 297-302 (1945)

6. Dubois, D., Prade, H.: A set-theoretic view of belief functions: Logical operations and approximations by fuzzy sets. Int J Gen Syst 12(3), 193-226 (1986) 
7. Dubois, D., Prade, H.: Consonant approximations of belief functions. Int J Approx Reason 4(5-6), 419-449 (1990)

8. Harmanec, D.: Faithful approximations of belief functions. Uncertain Artif Intell 15 (1990)

9. Helal, N., Pichon, F., Porumbel, D., Mercier, D., Lefevre, E.: The capacitated vehicle routing problem with evidential demands. Int J Approx Reason 95, 124-151 (2018)

10. Jaccard, P.: Étude comparative de la distribution florale dans une portion des alpes et des jura. Bulletin de la Société Vaudoise des Sciences Naturelles 37, 547-579 (1901)

11. Kallehauge, B.: Formulations and exact algorithms for the vehicle routing problem with time windows. Computers \& Operations Research 35(7), 2307-2330 (2008)

12. Kennes, R.: Computational aspects of the Möbius transformation of graphs. IEEE Trans. Syst. Man Cybern. Syst 22(2), 201-223 (1992)

13. Lowrance, J.D., Garvey, T.D., Wilson, T.M.S.N.: A framework for evidential reasoning systems. In: Kehler, T., al. (eds.) Proc. of AAAI' 86. vol. 2, pp. 896-903. AAAI (August 1994)

14. Petit-Renaud, S., Denœux, T.: Handling different forms of uncertainty in regression analysis: A fuzzy belief structure approach. In: Hunter, A., Parsons, S. (eds.) Symbolic and Quantitative Approaches to Reasoning and Uncertainty. pp. 340-351. Springer Berlin Heidelberg, Berlin, Heidelberg (1999)

15. Shafer, G.: A mathematical theory of evidence. Princeton University Press (1976)

16. Smets, P.: Belief functions: the disjunctive rule of combination and the generalized bayesian theorem. Int J Approx Reason 9, 1-35 (1993)

17. Tedjini, T., Afifi, S., Pichon, F., Lefevre, E.: A belief-constrained programming model for the VRPTW with evidential service and travel times. In: Proc. of $28 \mathrm{es}$ rencontres francophones sur la Logique Floue et ses Applications. pp. 217-224. Alès, France (2019)

18. Tessem, B.: Approximations for efficient computation in the theory of evidence. Artif. Intell 61(2), 315-329 (1993)

19. Voorbraak, F.: A computationally efficient approximation of Dempster-Shafer theory. Int $\mathbf{J}$ Man Mach Stud 30(5), 525-536 (1989)

20. Wilson, N.: A Monte-Carlo algorithm for Dempster-Shafer belief. In: D'Ambrosio, B., Smets, P., Bonissone, P. (eds.) Proc. of the 7th Conference on Uncertainty in AI, UAI'91. pp. 414-417. Morgan Kaufmann (1991)

21. Zwick, R., Carlstein, E., Budescu, D.V.: Measures of similarity among fuzzy concepts: A comparative analysis. Int J Approx Reason 1(2), 221 - 242 (1987) 